\title{
Computerised cognitive behavioural therapy for the treatment of depression in people with multiple sclerosis: external pilot trial
}

Cindy L Cooper ${ }^{1 *}$, Daniel Hind ${ }^{1}$, Glenys D Parry², Claire L Isaac ${ }^{3}$, Munyaradzi Dimairo ${ }^{1}$, Alicia O'Cathain², Anita Rose ${ }^{4}$, Jennifer V Freeman², Leonie Martin², Eva C Kaltenthaler², Anna Thake ${ }^{1}$ and Basil Sharrack ${ }^{5}$

\begin{abstract}
Background: People with multiple sclerosis (MS) are at high risk of depression. We undertook a pilot trial of computerised cognitive behavioural therapy (CCBT) for the treatment of depression in people with MS to test the feasibility of undertaking a full trial.

Methods: Participants with a diagnosis of MS and clinical levels of depression were recruited through out-patient clinics and postal screening questionnaires at two UK centres and randomised to CCBT or usual care. Clinical outcomes included the Beck Depression Inventory (BDI-II) and Multiple Sclerosis Impact Scale (MSIS-29) at baseline, 8 and 21 weeks. Feasibility outcomes included: recruitment rate; reasons for refusal, withdrawal and dropout; feasibility and acceptability of the proposed outcome measures; sample size estimation and variation in and preferences for service delivery.

Results: Twenty-four participants were recruited. The recruitment rate, calculated as the proportion of those invited to fill in a screening questionnaire who were consented into the trial, was 4.1\%. Recruitment through out-patient clinics was somewhat slower than through screening questionnaire mail-out but the overall recruitment yield was similar. Of the 12 patients in the CCBT arm, 9 (75\%) completed at least four, and 6 completed all 8 CCBT sessions. For completers, the median time (IQR) to complete all eight CCBT sessions was 15 (13 to 20) weeks. Participants expressed concern about the face validity of the Beck Depression Inventory II for the measurement of self-reported depression in people with MS. The MSIS-29 was the patient-reported outcome measure which participants felt best reflected their concerns. The estimated sample size for a full trial is between 180 and 390 participants. NHS partners were not delivering CCBT in community facilities and participants preferred to access CCBT at home, with no one expressing a preference for use of CCBT in an alternative location.
\end{abstract}

Conclusions: A definitive trial, with a recruitment window of one year, would require the participation of around 13 MS centres. This number of centres could be reduced by expanding the eligibility criteria to include either other neurological conditions or people with more severe depression. The MSIS-29 should be used as a patientimportant outcome measurement.

Trial registration: ISRCTN: ISRCTN81846800

\section{Background}

Multiple Sclerosis (MS) is a chronic immune mediated disease of the central nervous system which affects around $0.1 \%$ of Caucasians of north and central European ancestry [1]. MS is characterized by a variety of

\footnotetext{
* Correspondence: c.l.cooper@sheffield.ac.uk

${ }^{1}$ Clinical Trials Research Unit, ScHARR, University of Sheffield, Regent Court, 30 Regent Street, Sheffield, S1 4DA, UK

Full list of author information is available at the end of the article
}

symptoms including visual impairment, limb weakness, sensory disturbance, balance and postural problems, sphincter dysfunction, cognitive impairments, pain and fatigue [2]. In the majority of patients, the illness runs an initial relapsing remitting (RRMS) course characterized by episodes of acute neurological dysfunction followed by full or partial recovery, usually culminating in a secondary progressive (SPMS) course during which disability progresses gradually with or without

\section{() Biomed Central}


occasional relapses, minor remissions and plateaus [3]. Reports suggest that $50 \%$ of patients with MS experience major depression during their lifetime and up to $40 \%$ may have depression at any one time $[4,5]$. A Cochrane review, last updated in mid-2005, suggested there was some evidence that cognitive behavioural therapy (CBT) could be effective for the treatment of depression in people with MS [6].

The UK National Institute for Health and Clinical Excellence (NICE) recommends Cognitive Behavioural Therapy (CBT) as a treatment for mild to moderate depression, and there has been an increasing interest in its use to help people to remain in the workplace [7-9]. As therapist-led CBT is often inaccessible or prohibitively expensive, computerised CBT (CCBT) is recommended by NICE as part of a stepped-care model for the management of mild or moderate depression, typically delivered in a primary care setting $[10,11]$. NICE recommended one package in particular, Beating the Blues, for treatment of mild to moderate depression [10]. However, the management of mental health problems is often complicated by the co-presence of chronic physical illness, and treatments which have been validated in populations without chronic physical illness may not be appropriate or effective in such circumstances [12]. More recent NICE guidance recommends CCBT for the treatment of depression in people with chronic physical conditions whilst recommending that further randomised controlled trials of psychological interventions are undertaken for this population [13].

Because of the challenges inherent in evaluating complex interventions such as CCBT, the Medical Research Council's Complex Intervention Framework recommends a stepwise approach to evaluation, with pilot work preceding a full randomised controlled trial (RCT) [14]. The full study would be an RCT with economic evaluation alongside the trial to test the hypothesis that CCBT is clinically and cost-effective, compared to usual treatment, for the treatment of depression in people with MS. In a previous paper we reported qualitative research assessing the target group's views on the acceptability and appropriateness of CCBT [15]. In this paper we report the results of a pilot trial, designed to assess the feasibility of a research protocol for a multicentre trial and to estimate the variance of the treatment effect. The pilot trial did not attempt to provide evidence for the clinical effectiveness of CCBT for the treatment of depression in people with MS. Therefore, we have written up our findings in accordance with recommendations for CONSORT-modifications for reporting the results of pilot studies and pragmatic trials $[16,17]$.

The objectives of this pilot trial were to:
- identify recruitment rates and test practicalities of recruitment;

- identify withdrawal and dropout rates during treatment phase and three month follow up phase to estimate dropout over longer term;

- identify reasons for refusal, withdrawal and dropout;

- test feasibility and acceptability of the proposed outcome measures, including the client service receipt inventory (a questionnaire developed for the collection of information on costs, service utilisation and related matters) required for a full economic evaluation;

- identify effect size and its associated variability at end of treatment in order to calculate an appropriate sample size for the full trial;

- identify variation in use or delivery of the intervention both at home and in an external setting;

- identify rate of preference for use of intervention at home or elsewhere

\section{Methods}

\section{Participants and setting}

We invited 582 people diagnosed with MS to screen for a study evaluating an intervention for low mood by completing a screening questionnaire including prognostic and eligibility criteria and a copy of the Beck Depression Inventory II-21 Item (BDI) [18]. All invitees had relapsing remitting or secondary progressive MS, according to the modified McDonald criteria [19]. Invitations were made between October 2008 and July 2009: face-to-face by consultants in the Sheffield Teaching Hospitals NHS Foundation Trust MS clinic $(n=288)$; by the Sheffield MS nurses visiting patients $(\mathrm{n}=4)$; and, using ink-signed personalised letters mailed out from a neuropsychologist at Sheffield $(n=40)$ as well as from consultant neurologists at the Walton Centre for Neurology and Neurosurgery NHS Trust, Liverpool ( $\mathrm{n}=$ 250). At Sheffield potential participants were identified from clinic attenders, MS nurse patient visits and from patients with MS from the caseload of the neuropsychologist. At the Walton Centre potential participants were identified solely from the MS register. The MS services in both sites are specific entities and not general neurology clinics.

Respondents completed a second BDI before a screening interview at which they were screened for eligibility by a clinical psychologist. Inclusion criteria were: age of 18 years or above; BDI score of at least 14 on two consecutive occasions and no treatment from psychologist, psychotherapist or psychiatrist within the last three months. That is, the sample was rated as depressed, using the BDI, but was not necessarily self-identifying 
and treatment-seeking. Exclusion criteria were: poor English language skills or cognitive function (score of less than 24 on Mini Mental State Examination [20]); BDI score of at least 29 on two consecutive occasions; active suicidal ideas; current or life-time diagnosis of psychosis, organic mental disorder or substance dependency; Kurtzke Expanded Disability Status Scale (EDSS) score of 8.5 or above [21]). The EDSS is a tool for quantifying MS-related disability. Our threshold for ineligibility was intended to exclude those who were restricted to bed for much of the day and had only limited use of their arms, thus effectively precluding use of a computer mouse and keyboard. The clinical psychologist assessed participants for major depressive disorder using the Mini-International Neuropsychiatric Interview (MINI) [22], but this was not an eligibility criterion.

Potential participants who were identified as having severe depression (BDI score of at least 29) or active suicidal ideas during the screening process were contacted by the study clinical psychologists and briefly assessed to understand whether the reported symptoms were typical and advised to contact their GP if deemed necessary. Once recruited to the study, active monitoring of Beck Depression Inventory responses was the responsibility of the study manager who contacted the PCT mental health lead on identifying responses indicating active suicidal ideation. Responses were dealt with according to the primary care trust's individual protocols for handling suicidal ideation.

\section{Interventions}

After consent, we randomised participants either to CCBT using 'Beating the Blues ${ }^{(\mathbb{B}}$ (Ultrasis Ltd) or to usual care (Treatment as Usual - TAU). A central webbased randomisation service delivered by the Sheffield Clinical Trials Research Unit was used after patient eligibility had been confirmed. All TAU arm participants were offered the opportunity of accessing the intervention at the end of the trial. The study statisticians and principal investigator remained blinded to the treatment allocation codes until after the final analysis. Beating the Blues ${ }^{\circledR}$ consists of eight computer-interactive sessions, of approximately 50 minutes each in duration, designed to be taken weekly. Each session consists of a mix of cognitive and behavioural strategies, which the user customises to their individual problems. A client service receipt inventory was used at baseline, 8 weeks and 21 weeks to identify concomitant medication and service use in both arms: the research protocol did not manualise or restrict treatment as usual.

\section{Clinical outcomes}

The primary clinical endpoint was the mean change in self-reported symptoms of depression as measured on the BDI. Specifically, we measured the mean change in scores between the two arms as measured at baseline, 8 and 21 weeks (13 weeks post intervention). Secondary clinical endpoints, measured at the same time points, were: (1) MS specific Quality of Life (QoL), measured by the Multiple Sclerosis Impact Scale 29item (MSIS-29 [23]) questionnaire (physical and psychological components); general health related QoL, measured by the summary score for the Short Form-36 items (SF-36 [24]); overall improvement of depression severity and anxiety, measured by Patient Health Questionnaire-9 item (PHQ-9 [25]) and Generalised Anxiety Disorder 7-item (GAD-7 [26]) questionnaires respectively. A client service receipt inventory was used to capture concomitant use of health services and medication between follow-ups, to test collection of cost and activity data.

\section{Feasibility outcomes}

The pre-specified primary outcome was the recruitment rate, calculated as the proportion of those invited to fill in a screening questionnaire who were consented into the trial. The practicalities of recruitment were assessed descriptively (see next paragraph). Refusal, withdrawal and dropout from the study protocol were recorded. The feasibility, acceptability and appropriateness of data collection strategies were assessed descriptively and through item response rates. Variation in the delivery of CCBT by Primary Care Trusts (PCTs) was documented, in terms of whether they made provision for use at home and the level of support. Rates of preference for use of the intervention at home and withdrawal from treatment were calculated as a percentage of those randomised.

A short semi-structured telephone interview was conducted with all study participants on completion of CCBT during which they were asked how they felt about the processes of recruitment and randomisation, the location of CCBT, their reasons for dropping out or not completing (where appropriate) and the appropriateness of the quantitative outcome measures used in the study. The methods for data collection and analysis of this qualitative component have been reported previously [15].

\section{Feasibility criteria}

No criteria for evaluating the feasibility (in the sense of formal 'stop/go' criteria) of a definitive study were identified prospectively (see discussion).

\section{Sample size}

We used a sample size of 12 per group $(n=24)$, on the basis of feasibility and precision of estimates to be used to design the main study [27]. 


\section{Statistical methods}

Primary analysis was an Intention To Treat (ITT) analysis which analysed all 24 participants according to their randomised treatment assignment ignoring non compliance, protocol deviations and withdrawal. Statistical analysis was mainly descriptive [16] with outcome variability and patient response profiles analysed using summary measures at different time points. For this study, and in planning future studies it is most useful to present the absolute final values for individuals, rather than by how much individuals change from their initial baseline values as this allows comparison with population norms and other population groups. For these reasons we have presented the former rather than the latter as it provides the necessary information about both change and absolute values. Response rates on questionnaire items were high and last observation carried forward was used to impute the few missing items. Questionnaire response rates are given as a fraction of the total number of questions answered in a questionnaire among patients followed up on a specific visit relative to the total items. For example, the denominators for BDI and MSIS-29 are 21 and 29 questions respectively. Sample size calculation for a definitive trial was performed using an ANCOVA model, approximated standardised effect sizes corresponding to small, medium and large effect sizes, expected drop out rate, outcome variability and conservative correlation structure from this pilot study. All analysis was performed in Stata version 11.1.

\section{Ethical approval}

This study received ethics approval from Northern and Yorkshire Research Ethics Committee.

\section{Results}

\section{Recruitment rates and practicalities of recruitment}

Our initial aim was to recruit 24 participants between 22 October 2008 and 31 January 2009 (101 days; 7.1 participants/month) with candidates identified by three neurologists working in a single weekly specialist MS clinic (Sheffield Teaching Hospitals NHS Trust). We reached our recruitment target on 30 July 2009 (in 281 days; 2.6 participants/month) after adding a second centre (The Walton Centre NHS Foundation Trust, Liverpool). From the 288 invitation packs given out by Sheffield neurologists in the MS clinic, forms were completed and returned by $63(21.8 \%)$ candidates from which we randomised 13 in 281 days (4.5\% recruitment yield), or 1.4 participants per month. From the 250 recruitment packs sent out by post from The Walton Centre, forms were completed and returned by 64 (25.6\%) of recipients, from which 10 participants were randomised in 149 days (4.0\% recruitment yield), or two participants per month. Sheffield MS Nurses approached four patients and a further participant was identified and recruited in this way. A neuropsychologist, based in Sheffield also wrote to 40 patients, but none of the respondents were recruited. In the telephone interviews, no participants expressed any concern about the processes of recruitment and randomisation.

\section{Participant characteristics}

As this was a pilot study the sample size was small and by chance the random allocation of participants to the intervention and control groups resulted in imbalances between the groups at baseline with respect to gender, MS type and depression severity (Table 1).

\section{Refusal, withdrawal and dropout}

Of 582 patients invited to take part, 140 responded of whom 48 were not interested in participating, 68 were ineligible and 24 were randomised (Figure 1). Therefore the primary outcome, the recruitment rate, calculated as the proportion of those invited to fill in a screening questionnaire consented into the trial, was $4.1 \%$. Of those who responded but were ineligible: 41 suffered from minimal depression (BDI of 13 or less); 20 were suffering from severe depression (BDI of 29 of more on two separate occasions); five were already seeing a psychiatrist or similar; and two were living in non-participating PCTs. Two participants in each arm were lost to follow-up at 21 weeks post-randomisation. No patients were formally withdrawn from the study.

\section{Feasibility, acceptability, appropriateness of data collection strategies}

The collection of outcome data by postal questionnaire proved to be challenging, with multiple attempts to obtain data from many participants, particularly at later outcome assessment points. Nevertheless, primary outcome assessments were available for 21 (88\%) participants at 8 weeks from randomisation (or end of treatment if later) and $18(75 \%)$ participants at a further three months' follow-up. Furthermore, the completion rate for those questionnaires returned was high (Table 2).

The patient reported outcome measure which the participants felt best reflected their concerns was the MSIS29. During the telephone interviews, participants expressed concern about the face validity, for people with MS, of the questionnaires used for the measurement of self-reported depression, including the Beck Depression Inventory II. In particular they were concerned about the three symptoms, fatigue, sleep and concentration, which are also somatic symptoms of MS.

"[The questions are] not really appropriate for people with MS because they are things that you would have 
Table 1 Participant baseline characteristics

\begin{tabular}{|c|c|c|c|c|}
\hline Characteristic & Scoring & $\begin{array}{c}\mathrm{TAU}^{3} \\
(\mathrm{n}=12) \\
\mathrm{n}(\%)\end{array}$ & $\begin{array}{c}\mathrm{CCBT}^{4} \\
(\mathrm{n}=12) \\
\mathrm{n}(\%)\end{array}$ & $\begin{array}{c}\text { Total } \\
(\mathrm{N}=24) \\
\mathrm{n}(\%)\end{array}$ \\
\hline \multirow[t]{2}{*}{ Gender } & Male & $5(42 \%)$ & $1(8 \%)$ & $6(25 \%)$ \\
\hline & Female & $7(58 \%)$ & $11(92 \%)$ & $18(75 \%)$ \\
\hline \multicolumn{5}{|l|}{ Age (years) } \\
\hline & Min to $\max$ & 31 to 54 & 33 to 57 & 31 to 57 \\
\hline & Mean $(S D)^{1}$ & $42(7.0)$ & $48(7.7)$ & $45(7.9)$ \\
\hline & Median $(\mathrm{IQR})^{2}$ & 42 (37 to 47 ) & 49.8 (42 to 55$)$ & 45 (39 to 51$)$ \\
\hline \multicolumn{5}{|l|}{ EDSS score } \\
\hline & Min to max & 0.0 to 6.0 & 2.0 to 6.5 & 0.0 to 6.5 \\
\hline & Mean (SD) & $3.6(1.8)$ & $4.8(1.7)$ & $4.2(1.8)$ \\
\hline & Median (IQR) & 3.5 (2.8 to 5.0$)$ & 5.5 (3.5 to 6.3$)$ & 4.0 (3.0 to 6.0$)$ \\
\hline \multicolumn{5}{|l|}{ Centre } \\
\hline & Liverpool & $5(42 \%)$ & $5(42 \%)$ & $10(42 \%)$ \\
\hline & Yorkshire & $7(58 \%)$ & $7(58 \%)$ & $14(58 \%)$ \\
\hline \multicolumn{5}{|l|}{ MS type } \\
\hline & Relapsing-remitting & $12(100 \%)$ & $7(58 \%)$ & 19 (79\%) \\
\hline & Primary progressive & $0(0 \%)$ & $5(42 \%)$ & $5(21 \%)$ \\
\hline \multicolumn{5}{|c|}{ BDI total score (0 weeks) } \\
\hline & Min to max & 15 to 29 & 16 to 27 & 15.0 to 29.0 \\
\hline & Mean (SD) & $23(5.2)$ & $21(4.0)$ & $22(4.7)$ \\
\hline & Median (IQR) & 25 (19 to 29$)$ & 22 (17 to 25$)$ & 22 (18 to 26$)$ \\
\hline
\end{tabular}

${ }^{1}$ SD: standard deviation; ${ }^{2}$ IQR: inter-quartile range; ${ }^{3}$ TAU: Treatment as Usual; ${ }^{4}$ CCBT: Computerised Cognitive Behavioural Therapy.

anyway. They're not actually concerned with depression like not having good sleep. I mean, that's a very common problem with MS is you don't have refreshing sleep." (ID 271)

No issues were identified with the client service receipt inventory designed for the collection of cost and activity data. Identification of prognostic variables, particularly the EDSS and MS Type, from patient records was not always possible and had to be obtained directly from participants' neurologists for the purposes of the study in some cases.

\section{Variability of outcome measures and sample size estimation}

Patient profiles with respect to clinical outcomes are reported in Table 3 and Figures 2, 3, 4 and 5. The usual approach to estimating sample size is to base it on an important clinically significant change. However, the difficulties of assessing a clinically significant change in $\mathrm{BDI}$ in the MS population have been reported previously with no available guideline [28]. An alternative approach is to base the effect size on the estimated mean change in BDI between the two groups. We decided against doing this as baseline imbalances with respect to gender and MS-type (Table 1) would produce bias in the estimated intervention effect.
Instead we used approximate effect sizes expressed as a fraction of the pooled standard deviation of outcomes from this pilot which correspond to small, moderate and large standardised effect sizes. The standard deviations of the BDI, MSIS-29 psychological and physical domains were $8.3,8.2$ and 18.2 respectively, and variability of these outcome measures stratified by intervention group are shown in Table 4. Data at all time-points were included in these calculations and as such provide conservative estimates of the true standard deviation for these measures. The results related to the change in BDI and MSIS among complete cases are given in Table 5.

A mean difference of change in 5 points on the BDI corresponds to a moderate standardised effect size and equates to the upper estimate of the likely effect size [29]. To have $90 \%$ power to detect this effect size (assuming correlation between follow-up measurements of 0.75 , an attrition rate of $25 \%$ and a fixed type 1 error rate of $5 \%$ ) a total of 70 participants would be required. A difference between groups of 3 or 2 points would correspond to the lower estimates of the likely effect size and this would require a total sample size of 180 and 390 respectively at $90 \%$ power. The summary of sample size estimates at varying levels of power and effect size (expressed as an absolute value and as equivalent standardised effect size) are given in Table 6. 


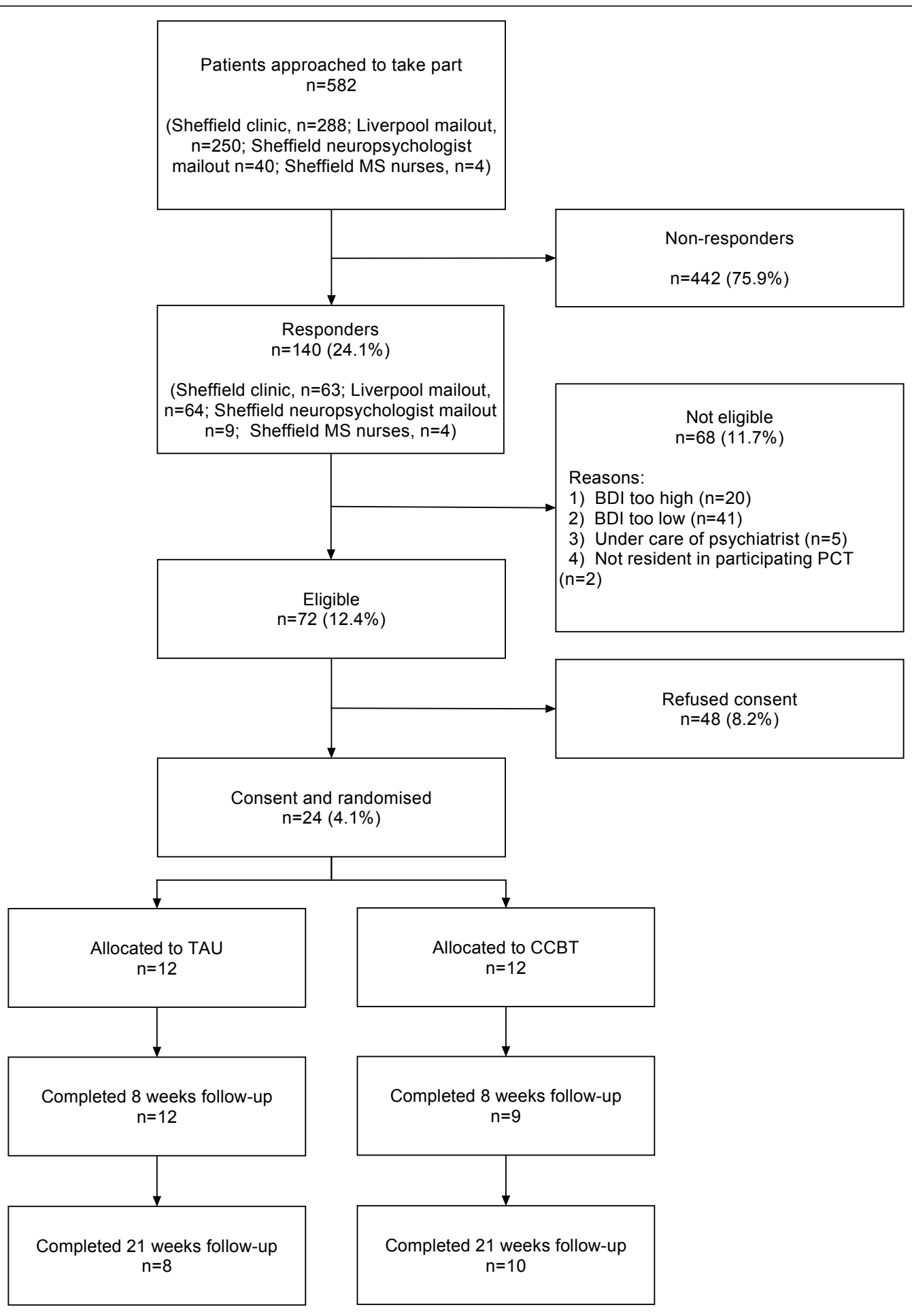

Figure 1 CONSORT diagram.

\section{Delivery of CCBT (home/external) and variation in delivery and use}

In the Sheffield region, Primary Care Trust (PCT) mental health care teams administered the CCBT, facilitating access and undertaking safety monitoring (PCTs were publicly funded organisations responsible for commissioning acute services for local populations). The CCBT service provided to trial participants was the routine 
Table 2 Questionnaire item response rates for available questionnaires

\begin{tabular}{|c|c|c|c|c|c|c|c|c|c|c|}
\hline \multirow[t]{2}{*}{ Questionnaire } & \multirow[t]{2}{*}{ Follow up Time point } & \multicolumn{3}{|c|}{ Number of participants } & \multicolumn{2}{|c|}{ TAU $\%$} & \multicolumn{2}{|c|}{ CCBT \% } & \multicolumn{2}{|c|}{ Overall \% } \\
\hline & & All & TAU & CCBT & Min to $\max$ & median & Min to $\max$ & median & Min to $\max$ & median \\
\hline \multirow[t]{3}{*}{$\mathrm{BDI}$} & 0 weeks & 24 & 12 & 12 & $95-100$ & 100 & $100-100$ & 100 & $95-100$ & 100 \\
\hline & 8 weeks & 21 & 12 & 9 & $95-100$ & 100 & $100-100$ & 100 & $95-100$ & 100 \\
\hline & 21 weeks & 18 & 8 & 10 & $91-100$ & 100 & $71-100$ & 100 & $71-100$ & 100 \\
\hline \multirow[t]{3}{*}{ MSIS-29 } & 0 weeks & 23 & 11 & 12 & $96-100$ & 100 & $100-100$ & 100 & $96-100$ & 100 \\
\hline & 8 weeks & 20 & 12 & 8 & $96-100$ & 100 & $100-100$ & 100 & $96-100$ & 100 \\
\hline & 21 weeks & 19 & 10 & 9 & $96-100$ & 100 & $96-100$ & 100 & $96-100$ & 100 \\
\hline \multirow[t]{3}{*}{ PHQ-9 } & 0 weeks & 23 & 11 & 12 & $90-100$ & 100 & $100-100$ & 100 & $90-100$ & 100 \\
\hline & 8 weeks & 21 & 12 & 9 & $90-100$ & 100 & $100-100$ & 100 & $90-100$ & 100 \\
\hline & 21 weeks & 18 & 8 & 10 & $90-100$ & 100 & $100-100$ & 100 & $90-100$ & 100 \\
\hline \multirow[t]{3}{*}{ GAD-7 } & 0 weeks & 23 & 11 & 12 & $71-100$ & 100 & $100-100$ & 100 & $71-100$ & 100 \\
\hline & 8 weeks & 21 & 12 & 9 & $100-100$ & 100 & $100-100$ & 100 & $100-100$ & 100 \\
\hline & 21 weeks & 18 & 9 & 9 & $100-100$ & 100 & 100-100 & 100 & $100-100$ & 100 \\
\hline \multirow[t]{3}{*}{ SF-36 } & 0 weeks & 23 & 11 & 12 & $91-100$ & 100 & $97-100$ & 100 & $91-100$ & 100 \\
\hline & 8 weeks & 21 & 12 & 9 & $66-100$ & 100 & $100-100$ & 100 & $66-100$ & 100 \\
\hline & 21 weeks & 19 & 9 & 10 & $94-100$ & 100 & $100-100$ & 100 & $94-100$ & 100 \\
\hline
\end{tabular}

service provided to all patients referred to the PCT mental health care teams. Only one out of the five participating PCTs around the Sheffield centre formally provided any kind of community facility for accessing CCBT; PCT staff warned that clients rarely used the facility and it was closed during the course of the study. Although four out of five participating PCTs were happy to arrange alternative provision for those who did not have Internet access at home, all our participants requested home use of CCBT. In the Liverpool region, a specialist neuropsychologist, based in the acute hospital facilitated access to and provided technical advice on CCBT, but did not provide additional therapeutic input. No provision other than home-use was offered or sought.

No PCT with whom we worked offered advice or support for defining problems on which to work or applying the CBT model to individual problems. The advice given was essentially technical, focusing on using the software. The PCTs delivering CCBT reported that they assumed that patients who were non-adherent and noncontactable beyond two weeks had discontinued treatment and made no further effort to contact them. Some but not all PCTs informed the patient's GP in such a situation. Preference for use at home amongst users in the study was universal with no-one expressing a preference for use of CCBT in an alternative location. Only one participant in the TAU arm took up the offer of access to CCBT at the end of the trial.

\section{Withdrawal from treatment}

Of the 12 patients in the CCBT arm, 9 (75\%) completed at least four CCBT sessions. This translated to a reasonably high compliance rate of CCBT although only six $(50 \%)$ of the patients in this arm completed all intended eight CCBT sessions. Of these six patients, the median

Table 3 Summary measures of patient profiles for primary and secondary outcomes stratified by intervention group

\begin{tabular}{lccccccccc}
\hline Outcome Measure & Follow -up & \multicolumn{3}{c}{ TAU } & \multicolumn{3}{c}{ CCBT } \\
\hline & & $\mathrm{n}$ & Mean(SD) & Median(IQR) & Min-Max & $\mathrm{n}$ & Mean(SD) & Median(IQR) & Min-Max \\
\cline { 3 - 9 } & Baseline & 12 & $23.3(5.2)$ & $24.5(19.0-28.5)$ & $15.0-29.0$ & 12 & $21.0(4.0)$ & $22.0(17.0-25.0)$ & $16.0-27.0$ \\
& 8 weeks & 12 & $22.1(9.1)$ & $20.0(18.0-25.0)$ & $7.0-44.0$ & 9 & $14.8(7.5)$ & $16.0(8.0-21.0)$ & $6.0-26.0$ \\
& 21 weeks & 8 & $24.4(11.4)$ & $21.5(18.0-33.0)$ & $8.0-42.0$ & 10 & $18.3(7.9)$ & $17.0(11.0-24.0)$ & $10.0-35.0$ \\
\hline MSIS-29 subscale & & & & & & & & \\
\hline Physical & Baseline & 11 & $60.7(20.9$ & $68.0(38.0-78.0)$ & $32.0-94.0$ & 12 & $62.8(14.2)$ & $65.0(50.5-71.5)$ & $42.0-87.0$ \\
& 8 weeks & 12 & $58.3(18.7)$ & $60.5(42.5-74.0)$ & $30.0-85.0$ & 8 & $58.8(19.0)$ & $65.5(40.0-71.0)$ & $33.0-84.0$ \\
& 21 weeks & 10 & $60.8(22.0)$ & $57.5(44.0-81.0)$ & $31.0-97.0$ & 9 & $55.8(18.0)$ & $58.0(43.0-65.0)$ & $27.0-82.0$ \\
\hline Psychological & Baseline & 11 & $27.3(8.2)$ & $28.0(20.0-37.0)$ & $15.0-38.0$ & 12 & $28.8(6.2)$ & $29.5(24.5-34.0)$ & $18.0-37.0$ \\
& 8 weeks & 12 & $26.8(8.9)$ & $27.5(20.0-33.0)$ & $14.0-43.0$ & 8 & $23.4(10.3$ & $25.5(13.0-29.5)$ & $11.0-40.0$ \\
& 21 weeks & 10 & $25.7(9.5)$ & $24.0(16.0-33.0)$ & $15.0-43.0$ & 9 & $24.4(6.6)$ & $22.0(21.0-31.0)$ & $17.0-34.0$ \\
\hline
\end{tabular}




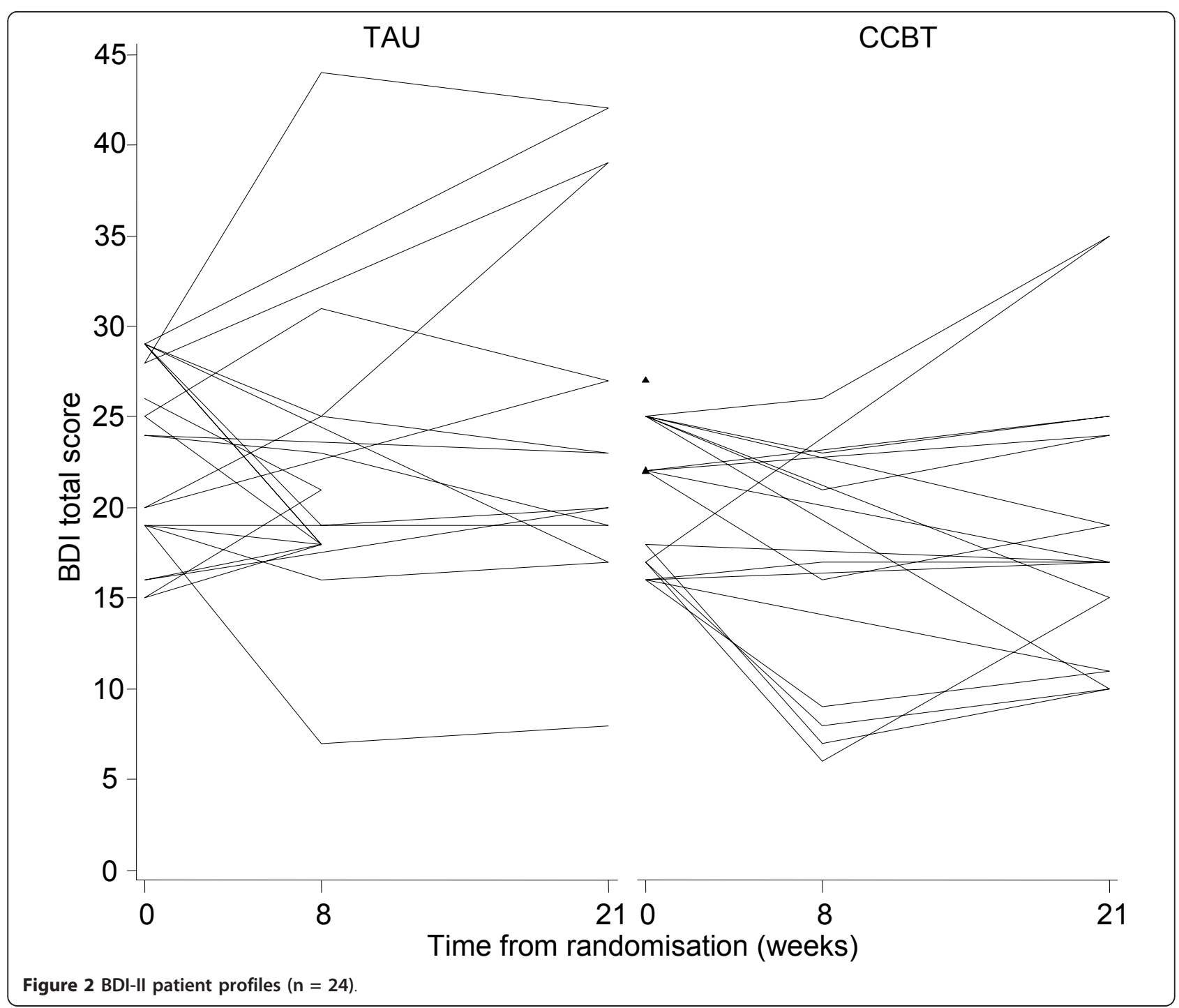

time (IQR) to complete all eight CCBT sessions was 15 (13 to 20) weeks against an intended eight weeks time frame. Only one person receiving CCBT formally requested discontinuation of treatment (after Session 6 of 8) citing time and lack of enthusiasm as reasons. Of the other non-completers, three also contributed qualitative data on their non-completion, one indicating lack of time, one that she no longer felt the need for treatment for her depression, and another citing computer hardware issues.

\section{Other concomitant service and medication use}

Four participants received some kind of talking therapy outside of the research protocol during the trial, one in the CCBT arm and three in the TAU arm. Thirteen participants received anti-depressants during the trial: seven in the CCBT arm; six in the TAU arm.

\section{Discussion}

Recruitment to this study was slower than expected. Dropouts and losses to follow-up were comparable with studies evaluating CCBT in non-MS populations: reported dropout rates for CCBT range from $0-75 \%$ (mean percentage dropout rate $32 \%$, SD 16.52), which is comparable to dropout rates for other psychological therapies [30]. Data collection by postal questionnaire was challenging. Participants found problems with the face validity of the depression inventories which include somatic symptoms of depression which are also symptoms of MS (the BDI-II and the PHQ-9), despite the fact that one has been psychometrically validated previously for use in people with MS by several research teams $[31,32]$. The difficulties associated with assessing mood in people with physical illness is recognised and has been discussed previously [ 13,33]. However, the 


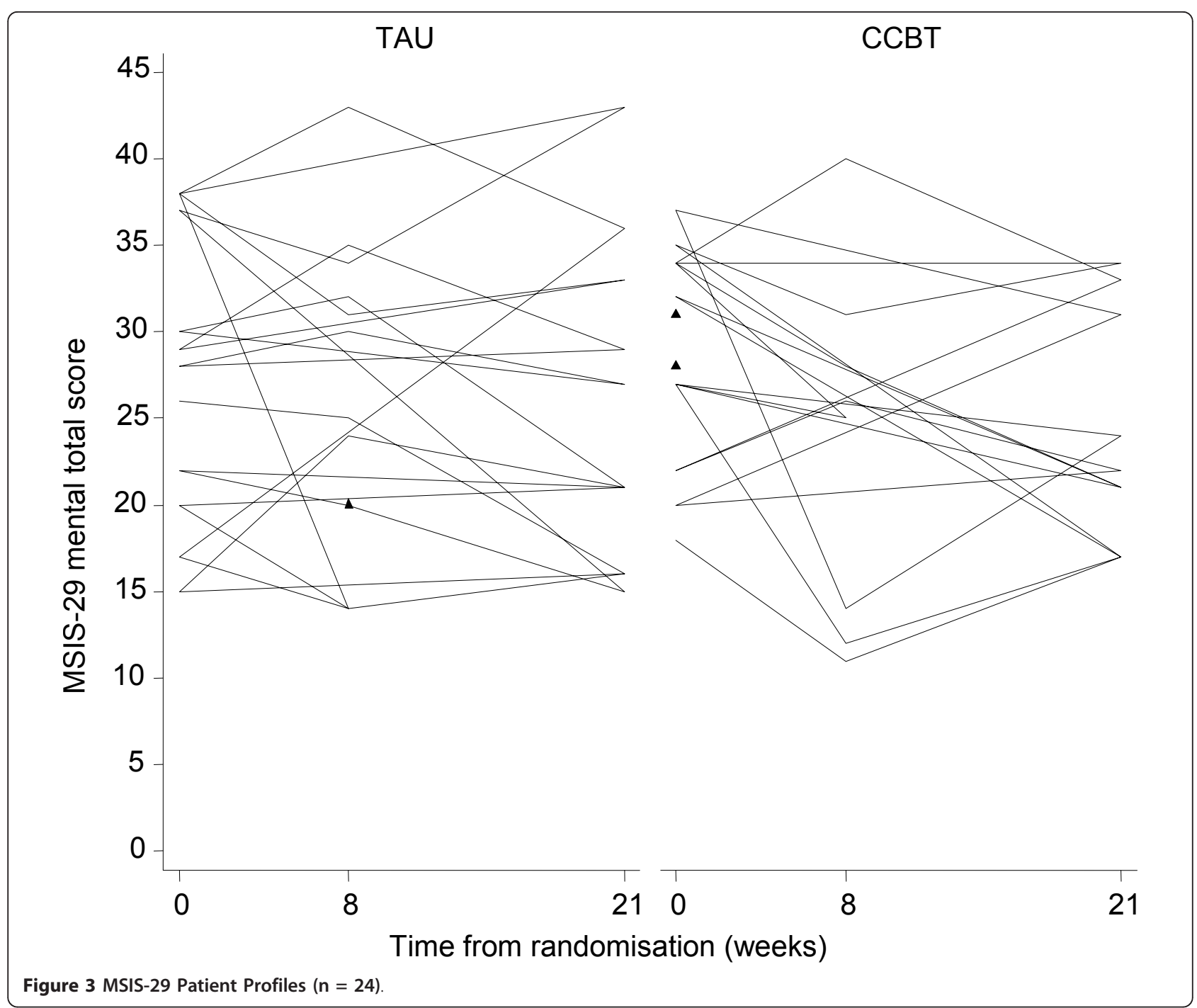

important point from this study is that participants expressed concerns about completing the measures and this may affect completion rates and validity in terms of consistency of participants' approach to responses throughout the course of the study. If a study is to evaluate impact on a patient-important outcome measure relevant to patients with MS then it should include the MSIS-29, which provides a measure of the physical and psychological impact of the condition from the patient's perspective [23]. This scale used on its own would reduce the ability to compare outcomes with other studies so it should be used in addition to general population scales such as the BDI, which has been used extensively in the general population.

A recent systematic Cochrane review identified a number of strategies proven to be effective in increasing response to postal questionnaires [34]. Fortunately, we had employed a few of these strategies including using a short, personalised (screening) questionnaire and including a second copy of the questionnaire at follow up. We also highlighted the University's involvement in the study. However, any future study could also employ other proven methods including using followup contact, use of stamped returned envelopes (as opposed to franked return envelopes), first class mailing and assurance of confidentiality in the letter of invitation.

Only a small percentage of people meeting the eligibility criteria for both MS and depression were successfully consented into the study. The population approached were not actively seeking treatment for mood-related symptoms and this may have been a contributing factor to the low consent rates. One other study has also identified that people with MS, while at higher risk of depression, are rarely treatment-seeking, at least in a primary care context [35]. 


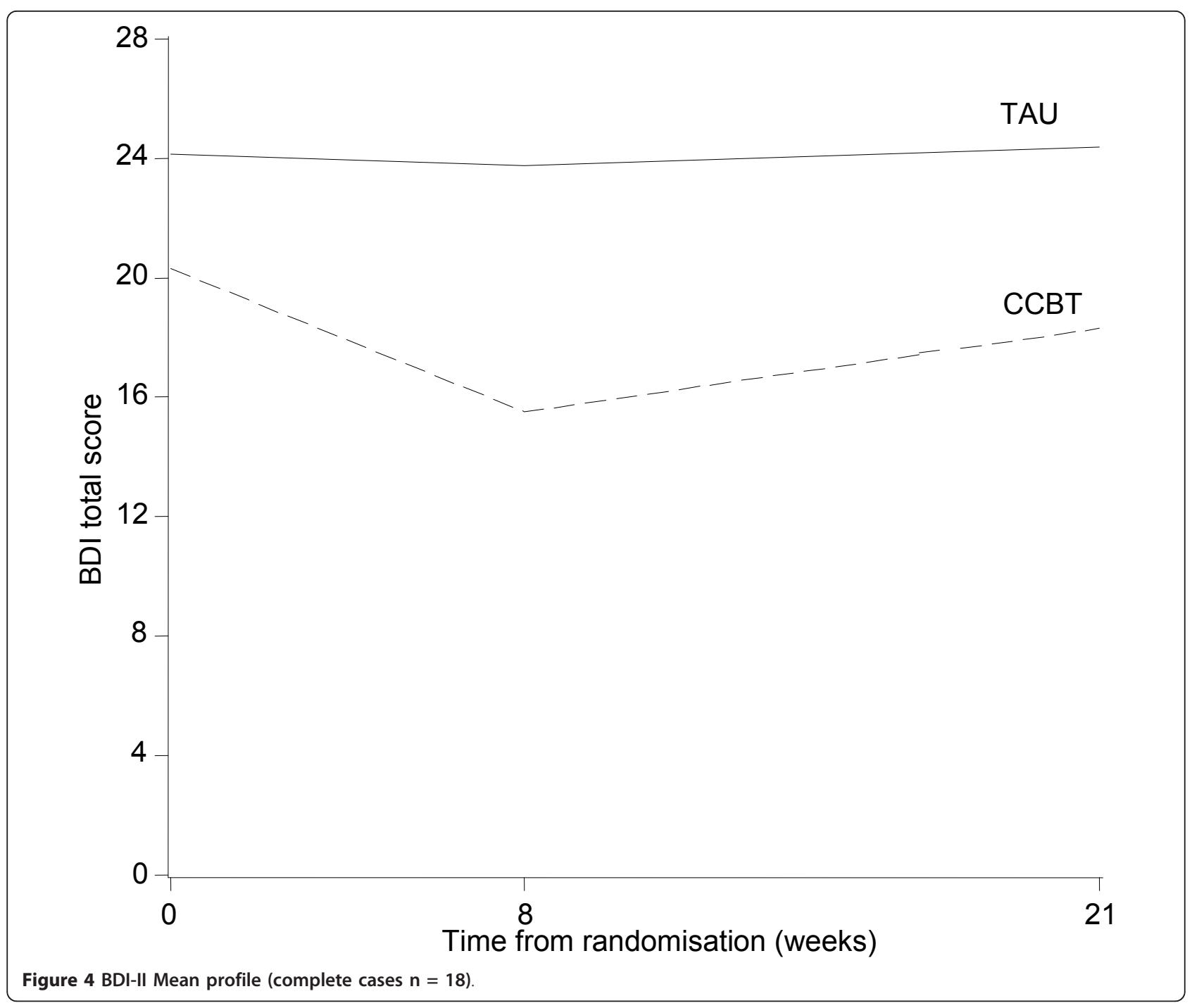

A limitation of this study is that it did not prospectively define formal 'stop/go' criteria for a definitive study, allowing researchers to assess the main study as not feasible, feasible with protocol modifications, or feasible without modifications (with or without close ongoing monitoring of protocol feasibility and implementation). This was recommended as good practice for pilot studies by Thabane and colleagues subsequent to the finalisation of our protocol and our grant award [16].

This pilot trial has demonstrated that recruitment of the required sample size for a full trial from the relevant population would be challenging as the numbers of participants recruited per site were very low and a high proportion of the prevalent cases had been approached in the recruitment period. Consequently, extending the recruitment period at particular centres would result in diminishing returns in terms of participant recruitment.
Postal screening via the Walton Centre resulted in 10 participants in 5 months, face to face recruitment at STH resulted in 14 participants in 9 months. From this evidence a full trial of 180 participants would require at least 13 sites, particularly considering that recruitment may be slower in a full-scale trial, where a team is reliant on other centres, than in a pilot study. This would require the participation of all the large, research active MS centres. Assuming 13 centres would be sufficient to recruit the required sample, the time to obtain all the necessary approvals and set up the sites would be considerable and 12 months at minimum should be allowed for this stage alone. In addition, time and resources should be allocated to allow for recruitment of additional sites if necessary

As we have tested recruitment using both identification of potential participants from an MS Register (Walton Centre) and through patient presentation at clinic 


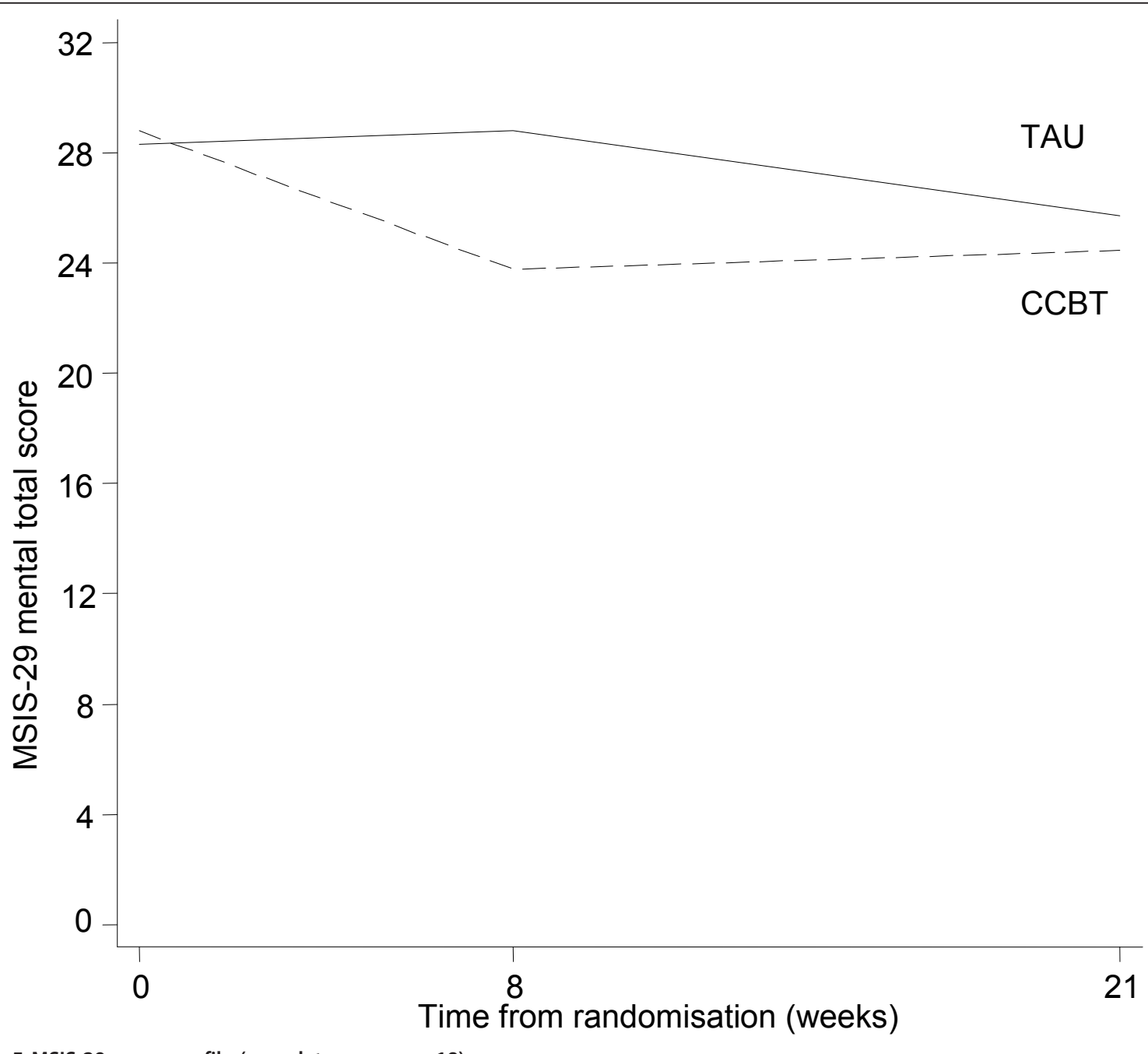

Figure 5 MSIS-29 mean profile (complete cases $n=18$ ).

(STH) either of these approaches could be utilised, so the service configuration would not be a limitation. However, the method of recruitment would also have to be considered carefully as we focused on a population who were screened for depression and not one which was actively seeking help for depression; this may have influenced the low uptake rate. In this regard, trialists may be in a difficult position: on the one hand, people

Table 4 Variability in outcomes (all time points) by intervention group

\begin{tabular}{llll}
\hline Outcome measure & \multicolumn{3}{c}{ Intervention group Mean (SD) } \\
\cline { 2 - 4 } & TAU & CCBT & All \\
\hline BDI & $24.1(9.0)$ & $18.0(6.7)$ & $20.7(8.3)$ \\
\hline MSIS-29 subscale & & \\
\hline Psychological & $26.6(8.6)$ & $25.9(7.7)$ & $26.3(8.2)$ \\
\hline Physical & $59.9(19.9)$ & $59.5(16.5)$ & $59.7(18.2)$ \\
\hline
\end{tabular}

Mean and SD for BDI and MSIS-29 subscales based on data at all time-points with MS show unusually low levels of help-seeking for their depression, making recruitment through systematic mass screening more appealing [35]; on the other hand, psychotherapeutic studies in which patients are recruited through systematic screening rather than their routine caregiver show significantly lower effect sizes

Table 5 Mean (SD) of change in primary and secondary outcomes relative to their baseline measurements.

\begin{tabular}{llllll}
\hline Outcome measure & Follow-up & \multicolumn{2}{c}{ TAU } & & \multicolumn{1}{c}{ CCBT } \\
\hline & & $\mathrm{N}$ & Mean (SD) & $\mathrm{n}$ & Mean (SD) \\
\cline { 3 - 6 } & 8 weeks & 12 & $-1.17(8.1)$ & 9 & $-5.33(4.7)$ \\
& 21 weeks & 8 & $0.25(8.8)$ & 10 & $-2.00(5.1)$ \\
\hline MSIS-29 subscale & & & & & \\
\hline Physical & 8 weeks & 11 & $-1.82(7.8)$ & 8 & $-7.00(12.9)$ \\
& 21 weeks & 10 & $-2.20(11.0)$ & 9 & $-7.89(12.3)$ \\
\hline Psychological & 8 weeks & 11 & $0.18(5.1)$ & 8 & $-5.88(9.5)$ \\
& 21 weeks & 10 & $-2.60(5.6)$ & 9 & $-4.33(8.0)$ \\
\hline
\end{tabular}


Table 6 Sample size estimates for a definitive trial stratified by outcome measure, power and effect size

\begin{tabular}{|c|c|c|c|c|c|}
\hline \multirow{2}{*}{$\begin{array}{l}\text { Outcome } \\
\text { measure }\end{array}$} & \multirow{2}{*}{$\begin{array}{l}\text { Power } \\
\text { (\%) }\end{array}$} & \multicolumn{2}{|c|}{ Effect size } & \multirow{2}{*}{$\begin{array}{l}\text { Sample Size per } \\
\text { group }\end{array}$} & \multirow{2}{*}{$\begin{array}{c}\text { Total sample size (attrition } \\
\text { adjusted) }\end{array}$} \\
\hline & & $\begin{array}{l}\text { Approximate mean } \\
\text { difference }\end{array}$ & Standardised & & \\
\hline \multirow[t]{6}{*}{$\overline{\mathrm{BDI}}$} & 90 & 5 & 0.6 & 35 & 70 \\
\hline & & 3 & 0.3 & 90 & 180 \\
\hline & & 2 & 0.2 & 195 & 390 \\
\hline & 80 & 5 & 0.6 & 25 & 50 \\
\hline & & 3 & 0.3 & 65 & 130 \\
\hline & & 2 & 0.2 & 145 & 290 \\
\hline \multicolumn{6}{|c|}{ MSIS-29 subscale } \\
\hline \multirow[t]{4}{*}{ Psychological } & 90 & 3 & 0.3 & 95 & 190 \\
\hline & & 2 & 0.2 & 220 & 410 \\
\hline & 80 & 3 & 0.3 & 70 & 140 \\
\hline & & 2 & 0.2 & 155 & 310 \\
\hline \multirow[t]{4}{*}{ Physical } & 90 & 6 & 0.3 & 115 & 230 \\
\hline & & 4 & 0.2 & 260 & 520 \\
\hline & 80 & 6 & 0.3 & 85 & 170 \\
\hline & & 4 & 0.2 & 195 & 390 \\
\hline
\end{tabular}

and the results of such a trial have poorer external validity [36]. Considering all these factors for a future full trial it may be better to extend the participant population to include people with other neurological conditions or even chronic conditions more widely. In addition, the eligibility criteria could be extended to include people with severe depression as in the ongoing Randomised Evaluation of the Effectiveness and Acceptability of Computerised Therapy (REEACT) Trial (ISRCTN 91947481). (Twenty respondents to our screening questionnaire, who might otherwise have been randomised, were excluded on the basis of experiencing severe depression at two measurement points). However, the sample size required to detect a difference may vary as our estimations are not based on this population.

It would be essential to maximise participant followup. A range of contact methods should be sought at the outset including mobile phone numbers and e-mail addresses. Sufficient resources would be required to ensure persistent followup of non-responders.

A further consideration for any future trial relates to the study design. The design of this study is described as CCBT vs TAU as the participants in the CCBT arm received $\mathrm{CCBT}$ in addition to any care, medication or services they would have received if they had not been part of the study. The TAU arm could be criticised as not reflecting usual treatment for depression but being a no-treatment arm (or TAU for MS) due to low levels of help-seeking for depression by people with MS [35]. An alternative approach would be to assess all participants for depression and to agree clinical management prior to randomisation. Those receiving TAU would therefore be offered appropriate treatment for depression, those in the treatment arm would receive CCBT in addition. This would also ensure that screened patients who do not meet the eligibility criteria on the basis of severity of depression would receive the appropriate clinical care.

The time taken for participants to complete the study intervention (13 to 20 weeks from randomisation) was longer than expected or than recommended in the product manual, due to the poor adherence of participants with the weekly schedule. Adherence to treatment programme timescales is difficult to guarantee where treatment is delivered by local NHS services and not delivered in ideal conditions by the trial team. Outcome assessment time-points of future studies should be fixed relative to the point of randomisation and not the projected end of the study intervention, as treatment programmes often over-run. This minimises discrepancies between the timepoints at which the treatment and control participants are followed up. Fixed timepoint outcome assessment should be supported by systems to minimise mis-timed outcome measurements, for instance automated follow-up reminders, pre-scheduled follow-up appointments and requesting alternative and preferred modes of contact (landline, mobile, text, email).

Participants in our previous related research reported that they would value more support in defining their problems and goals [15]. Since this research began, Spek and colleagues have demonstrated that CCBT programmes are markedly more effective when delivered with therapist support. However, in the PCTs with whom we worked, therapist support was only available 
where issues remain unresolved after a complete course of CCBT, although more recently, through the 'Improving Access to Psychological Therapies' service, the support of a psychological wellbeing practitioner within primary care should be available within the stepped care model recommended by NICE [8]. Whether such support is in fact available is likely to vary between sites [7]. Any future research protocol would have to balance the desire to provide and evaluate an optimised intervention (CCBT with concurrent therapist support) against the likelihood of therapist-supported CCBT becoming the norm in the UK healthcare setting. In either case, study teams should report any standardisation or variation in the implementation of the intervention (for instance in the level of therapist support) between study sites in line with the CONSORT modification proposed by Zwarenstein and colleagues [17]. We would not recommend evaluating the delivery of CCBT in community facilities as our experience here has shown that many NHS partners are not delivering it in this way and participants overwhelmingly chose to access CCBT at home.

More successful models of delivering CCBT and implementing future interventional research might be through either (a) the secondary care multi-disciplinary neurology teams (see for example work by Moss-Morris and colleagues [37]) or (b) Psychological Wellbeing Practitioners based in primary care trained by clinical neuropsychologists in the issues they need to know to support CCBT use in patients with MS. The commercial company which produces Beating the Blues ${ }^{\circledR}$ (Ultrasis) has indicated that it intends to address the issues of appropriateness and acceptability of Beating the Blues ${ }^{\mathbb{B}}$ to people with MS identified in our previous work [14] in future product developments. The choice of CCBT to be used in any future full trial should take into consideration whether these issues have been addressed and the range of alternative CCBT packages which may be available in the future.

\section{Conclusions}

The results of this pilot study demonstrate that, if the intended full trial of the clinical and cost-effectiveness of CCBT for depression in people with MS were to proceed, a number of amendments to the pilot trial protocol would be required. A definitive RCT, with a recruitment window of one year, would require the participation of around 13 UK MS centres. This number could be reduced by expanding the eligibility criteria to include either other neurological conditions or people with more severe depression. The design of the definitive study will need to consider whether to optimise the delivery of CCBT through the provision of therapist support depending on the likelihood of this being deliverable in routine practice. Participants should access
CCBT at home in line with patient-preference and because NHS partners are not typically delivering it in community facilities. Finally, the MSIS-29 should be used as a patient-important outcome measurement.

\section{Acknowledgements}

We thank the MS Society for funding the study and 24 generous participants who offered us their time. We would like to acknowledge the support of the following clinicians who facilitated participant recruitment to the study: Dr Mike Boggild, Consultant Neurologist at the Walton Centre NHS Trust, Liverpool; Drs Sian Price and Steve Howell, Consultant Neurologists, Diane Watts and the MS Specialist Nursing Team at Sheffield Teaching Hospitals NHS Foundation Trust. We would also like to acknowledge the support of the staff at the PCTs: Simon Bennett (Sheffield PCT); Linda Matthews, Liz Holdsworth, Matthew Swan (Barnsley PCT); Dawn White (Doncaster PCT); Georgina Horobin (Derbyshire County PCT); Sue Schmitz-Butler and Edward Randerson (Rotherham PCT). We would also like to acknowledge the technical, procedural and moral support of Kathy Griffiths (Australian National University), Charlie Martin and Despina Learmonth (Ultrasis). The following members of the Trial Steering Committee also provided invaluable expertise and advice throughout: Karen Beck (our study administrator); Professors John Brazier and Jon Nicholl of the University of Sheffield; Ray Stonehill, MS Society Kingston on Thames Branch.

\section{Author details}

${ }^{1}$ Clinical Trials Research Unit, ScHARR, University of Sheffield, Regent Court, 30 Regent Street, Sheffield, S1 4DA, UK. ${ }^{2}$ SCHARR, University of Sheffield, Regent Court, 30 Regent Street, Sheffield, S1 4DA, UK. ${ }^{3}$ Clinical Psychology Unit, Department of Psychology, The University of Sheffield, Western Bank, Sheffield S10 2TP, UK. ${ }^{4}$ The Walton Centre of Neurology \& Neurosurgery, Liverpool L9 7LJ, UK. 'Department of Neurology, Royal Hallamshire Hospital, Sheffield S10 2JF, UK.

\section{Authors' contributions}

CC was the chief investigator and drafted the manuscript. CC and GP conceived of the study. CC, GP, JF, BS, AO, Cl, AR and EK designed the study. $\mathrm{DH}$ was the study co-coordinator, conducted qualitative research interviews and helped to draft the manuscript. The trial management group were $\mathrm{CC}, \mathrm{DH}, \mathrm{AO}, \mathrm{Cl}, \mathrm{AR}, \mathrm{LM}$ and all, together with $\mathrm{BS}$, helped to implement the study on a day-to-day basis. MD and JF were the study statisticians and contributed to the statistical analysis plan. MD undertook the statistical analysis and helped to draft the manuscript. LM provided a perspective on living with multiple sclerosis. AT conducted qualitative research interviews. All authors read, commented on and approved the final manuscript.

\section{Competing interests}

The authors declare that they have no competing interests.

Received: 3 June 2011 Accepted: 14 December 2011

Published: 14 December 2011

\section{References}

1. Sadovnick AD, Dyment DA, Ebers GC, Risch NJ, the Canadian Collaborative Study Group: Evidence for genetic basis of multiple sclerosis. The Lancet 1996, 347:1728-1730.

2. Sharrack B, Hughes RAC: Scale development and Guy's Neurological Disability Scale. Journal of Neurology 1999, 246:226.

3. Confavreux C, Compston A: The natural history of multiple sclerosis. In McAlpine's Multiple Sclerosis.. 4 edition. Edited by: Compston A. London: Elsevier; 2006:183-272

4. Benito-León J, Manuel Morales J, Rivera-Navarro J, Mitchell AJ: A review about the impact of multiple sclerosis on health-related quality of life. Disability \& Rehabilitation 2003, 25:1291-1303.

5. Siegert RJ, Abernethy DA: Depression in multiple sclerosis: a review. $J$ Neurol Neurosurg Psychiatry 2005, 76:469-475.

6. Thomas PW, Thomas S, Hillier C, Galvin K, Baker R: Psychological interventions for multiple sclerosis. Cochrane Database of Systematic Reviews 2006, 1, Art. No.: CD004431 DOI: 10.1002/14651858.CD004431. pub2. 
7. Layard R: The case for psychological treatment centres. BMJ 2006, 332:1030-1032.

8. National Collaborating Centre for Mental Health: Depression: Management of depression in primary and secondary care (National Clinical Practice Guideline Number 23). London, National Institute for Health and Clinical Excellence; 2008, Ref Type: Report.

9. Seymour L, Grove B: Workplace Interventions for People with Common Mental Health Problems. London, British Occupational Health Research Foundation; 2005, Ref Type: Report.

10. Care Services Improvement Partnership, Primary Care Services for Depression: A guide to best practice. London, Department of Health; 2006, Ref Type: Report.

11. National Institute for Health and Clinical Excellence, Technology Appraisal 97: Computerised cognitive behavioural therapy for depression and anxiety. Review of Technology Appraisal 51 London, NICE; 2006, Ref Type: Report.

12. Creed F: Assessing depression in the context of physical illness. In Perspectives in Psychiatry Volume 6: Depression and Physical IIIness. Edited by: Robertson MM, Katona CLE. Baffins Lane, England: John Wiley; 1997:3-19.

13. National Collaborating Centre for Mental Health: Depression in adults with a chronic physical health problem: Treatment and management. NICE Clinical Guideline 91 London, National Institute for Health and Clinical Excellence; 2009.

14. Campbell NC, Murray E, Darbyshire J, Emery J, Farmer A, Griffiths F, Guthrie B, Lester H, Wilson P, Kinmouth AL: Designing and evaluating complex interventions to improve health care. BMJ 2007, 334:455-459.

15. Hind D, O'Cathain A, Cooper CL, Parry GD, Isaac CL, Rose A, Martin L, Lharrack B: The acceptability of computerised cognitive behavioural therapy for the treatment of depression in people with chronic physical disease: A qualitative study of people with multiple sclerosis. Psychology \& Health 2010, 25:699-712.

16. Thabane L, Ma J, Chu R, Cheng J, Ismaila A, Rios L, Robson R, Thabane M, Giangregorio L, Loldsmith $\mathrm{CH}$ : A tutorial on pilot studies: the what, why and how. BMC Medical Research Methodology 2010, 10:1.

17. Zwarenstein M, Treweek S, Gagnier JJ, Altman DG, Tunis S, Haynes B, Oxman DA, Moher D: Improving the reporting of pragmatic trials: an extension of the CONSORT statement. BMJ 337.

18. Beck AT, Steer RA, Ball R, Ranieri WF: Comparison of Beck Depression Inventories-IA and-II in Psychiatric Outpatients. Journal of Personality Assessment 1996, 67:588-597.

19. Polman $\mathrm{CH}$, Reingold SC, Edan G, Filippi M, Hartung H-P, Kappos L, Lublin F, Metz LM, McFarland HF, O'Connor PW, Sandberg-Wollheim M, Thompson AJ, Weinshenker BG, Wolinsky JS: Diagnostic criteria for multiple sclerosis: 2005 revisions to the IdquoMcDonald Criteriardquo. Annals of Neurology 2005, 58:840-846.

20. Folstein MF, Folstein SE, McHugh PR: "Mini-mental state": A practical method for grading the cognitive state of patients for the clinician. Journal of Psychiatric Research 1975, 12:189-198.

21. Kurtzke JF: Rating neurologic impairment in multiple sclerosis: An expanded disability status scale (EDSS). Neurology 1983, 33:1444-1452.

22. Sheehan DV, Lecrubier $Y$, Sheehan $K H$, Amorim $P$, Janavs J, Weiller $E$, Hergueta T, Baker R, Dunbar GC: The Mini-International Neuropsychiatric Interview (M.I.N.I.): the development and validation of a structured diagnostic psychiatric interview for DSM-IV and ICD-10. J Clin Psychiatry 1998, 59:22-33.

23. Hobart JC, Riazi A, Lamping D, Fitzpatrick R, Thompson AJ: Hobart JCImproving the evaluation of therapeutic interventions in multiple sclerosis: development of a patient-based measure of outcome. Health Technol Assess 2004, 8:iii, 1-48.

24. Brazier JE, Harper R, Jones NM, O'Cathain A, Thomas KJ, Usherwood T, Westlake L: Validating the SF-36 health survey questionnaire: new outcome measure for primary care. BMJ 1992, 305:160-164.

25. Spitzer RL, Kroenke K, Williams JBW, and the Patient Health Questionnaire Primary Care Study Group: Validation and Utility of a Self-report Version of PRIME-MD: The PHQ Primary Care Study. JAMA: The Journal of the American Medical Association 1999, 282:1737-1744.

26. Spitzer RL, Kroenke K, Williams JBW, Lowe B: A Brief Measure for Assessing Generalized Anxiety Disorder: The GAD-7. Arch Intern Med 2006, 166:1092-1097.

27. Julious SA: Sample Size Of 12 Per Group Rule Of Thumb For A Pilot Study. Pharmaceutical Statistics 2005, 4:287-291.
28. Mohr DC, Boudewyn AC, Goodkin DE, Bostrom A, Epstein L: Comparative outcomes for individual cognitive-behavior therapy, supportiveexpressive group psychotherapy, and sertraline for the treatment of depression in multiple sclerosis. J Consult Clin Psychol 2001, 69:942-949.

29. Cohen J: Statistical power analysis and for the behavioral sciences Hillsdale (NJ): Erlbaum; 1988.

30. Kaltenthaler E, Sutcliffe P, Parry G, Beverley C, Rees A, Ferriter M: The acceptability to patients of computerized cognitive behaviour therapy for depression: a systematic review. Psychological Medicine 2008, 38:1521-1530.

31. Aikens JE, Reinecke MA, Pliskin NH, Fischer JS, Wiebe JS, McCracken LM, Maylor $J$ : Assessing depressive symptoms in multiple sclerosis: is it necessary to omit items from the original Beck Depression Inventory? Journal of Behavioral Medicine 1999, 22(2):127-42.

32. Moran PJ, Mohr DC: The Validity of Beck Depression Inventory and Hamilton Rating Scale for Depression Items in the Assessment of Depression Among Patients with Multiple Sclerosis. Journal of Behavioral Medicine 2005, 28:35-41.

33. House A: Mood Disorders in the physically ill. Problems of definition and measurement. Journal of Psychosomatic Research 1988, 32(4/5):345-353.

34. Edwards PJ, Roberts I, Clarke MJ, Diguiseppi C, Wentz R, Kwan I, Cooper R, Felix LM, Pratap S: Methods to increase response to postal and electronic questionnaires. Cochrane Database Systematic Review Database 2009, 3.

35. Sollom AC, Kneebone II: Treatment of depression in people who have multiple sclerosis. Multiple Sclerosis 2007, 13:632-635.

36. Cuijpers P, van Straten A, van Schaik A, Andersson G: Psychological treatment of depression in primary care: a meta-analysis. British Journal of General Practice 59:e51-e60.

37. Moss-Morris R, Dennison L, Yardley L, Landau S, Roche S, McCrone P, Chalder T: Protocol for the saMS trial (supportive adjustment for multiple sclerosis): a randomized controlled trial comparing cognitive behavioral therapy to supportive listening for adjustment to multiple sclerosis. BMC Neurology 2009, 9:45.

doi:10.1186/1745-6215-12-259

Cite this article as: Cooper et al: Computerised cognitive behavioural therapy for the treatment of depression in people with multiple sclerosis: external pilot trial. Trials 2011 12:259.

\section{Submit your next manuscript to BioMed Central and take full advantage of:}

- Convenient online submission

- Thorough peer review

- No space constraints or color figure charges

- Immediate publication on acceptance

- Inclusion in PubMed, CAS, Scopus and Google Scholar

- Research which is freely available for redistribution

Submit your manuscript at www.biomedcentral.com/submit
C Biomed Central 